\title{
Research on harmonic current suppression of three-phase four-wire power conversion system
}

\author{
Hongyang Qing, Hao He, Wenqing Nie, Xiaohuan Wang, and Chunjiang Zhang* \\ Yanshan University, School of Electrical Engineering, Qinhuangdao, China
}

\begin{abstract}
The harmonic current of the three-phase four-wire gridconnected power conversion system (PCS) will be increased due to gridside harmonics and neutral loop. To solve these problems, the three-phase four-wire I-type three-level converter is applied as the topology in this paper to study the harmonic current suppression of the grid-connected PCS. A compound control strategy with the proportional integral (PI) and repetitive controller in parallel is presented and the interaction between the parameters of the compound controller is analyzed. The comparative research is carried out from the theoretical perspective of model analysis, and a controller optimization scheme is proposed. Finally, under the grid and neutral current waveform quality of simulation and experiment, the correctness and effectiveness of the proposed scheme is verified.
\end{abstract}

Keywords: Three-phase four-wire; Power conversion system; Compound control; Harmonic suppression.

\section{Introduction}

Distributed generation (DG) topologies and operation modes are diverse and need to meet the requirements of the power grid. The PCS is the important device of DG, not only its own stable operation and various functional requirements of DG need to be ensured, but also the three-phase imbalance of the power grid and single-phase load energy issues need to be considered. Thus, the PCS is mainly a three-phase four-wire form with the zerosequence current channel [1]. The non-linearity of high-density power electronic equipment leads to a large number of harmonic sources in the power grid and the increase of grid voltage harmonics [2], thus the grid current THD of PCS will be increased under the clamping action of the grid. The harmonics will be injected into the direct current (DC) side of the converter due to the neutral loop current, which contribute to the aggravating of adverse impact on the quality of the grid current. Excessive harmonic current will not only increase the loss and noise of power equipment, but also cause system failure in serious cases, threatening the safe operation of power system. According to the regulation IEC $61000-3-4$, the grid current THD of 5\% must not be exceeded [3]. Therefore, it is significant to suppress the harmonic current of the grid-connected PCS.

At present, there have been extensively studies on the related methods of harmonic control [4]. The resonance controller was applied to control harmonics in [5], which has a

\footnotetext{
*Corresponding author: zhangcj@ysu.edu.cn
} 
certain effect on improving the current quality. However, multiple resonant controllers need to be connected in parallel when there are a large number of different order harmonic currents, which will increase the complexity of control and decrease system stability. The repetitive control has been commonly used as the main control method to solve the influence of periodic harmonic disturbances because of its better steady-state performance and facile implementation [6]. But the conventional repetitive controller can hardly achieve a fast dynamic performance and requires the assistance of other controllers [7]. The double repetitive controller was employed to improve the dynamic performance of harmonic compensation in [8], but the compensation ability was still insufficient in the area where the harmonic current changes significantly, and the complexity of the algorithm was increased. The output current quality was improved by combining PI and repetitive controller in [9]. This compound control combines the advantages of the two controllers. While accurately tracking the rated value, it can also periodically compensate for harmonics and reduce the grid-connected current THD. However, the control object with accurate mathematical model is required for the combination of PI and repetitive controller, and reasonable adjustment of parameters is needed to obtain optimal control. An optimization model to coordinate the control of harmonic compensation was proposed which enhanced the harmonic control capability of the energy storage system in [10]. It demonstrated that it is also important to utilize the model to investigate harmonic suppression.

\section{System model}

The three-phase four-wire I-type three-level topology of grid-connected PCS is shown in Fig. $1 . U_{\mathrm{dc}}$ is the DC voltage of the energy storage system, $C_{\mathrm{d} 1}$ and $C_{\mathrm{d} 2}$ are the DC voltagedivider capacitors, $S_{\mathrm{x} 1}, S_{\mathrm{x} 2}, S_{\mathrm{x} 3}, S_{\mathrm{x} 4}(x=a, b, c)$ are power transistors, $L_{\mathrm{f}}$ and $R_{\mathrm{f}}$ are the filter inductance and its parasitic resistance, $C_{\mathrm{f}}$ is the filter capacitor, $L_{\mathrm{n}}$ is the equivalent line inductor of neutral loop, $i_{\mathrm{ox}}$ represents the grid current, $i_{\mathrm{Lx}}$ represents the filter inductance current and $U_{\mathrm{A}}, U_{\mathrm{B}}, U_{\mathrm{C}}$ represents the three-phase power grid. The neutral wire is connected through the neutral point $N$ of the power grid, the neutral point $N_{\mathrm{C}}$ of the filter capacitor and the midpoint $O$ of the DC side voltage divider capacitor.

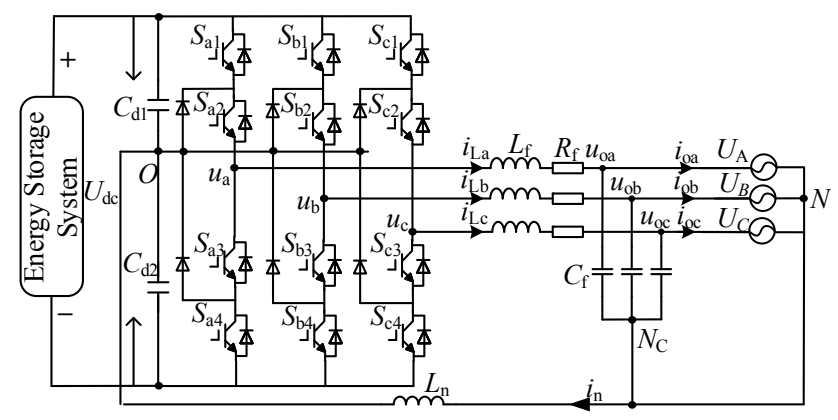

Fig. 1. The three-phase four-wire I-type three-level topology of grid-connected PCS.

The switch function is be defined as follows:

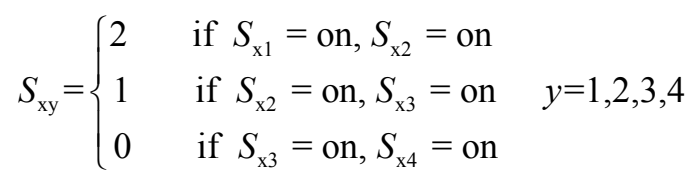

The voltage between the bridge arm of each phase and the neutral point of the grid can be written as: 


$$
u_{x}=\left(S_{\mathrm{xy}}-1\right) U_{\mathrm{dc}}
$$

The variables of (1) and Fig. 1 are transformed into $d q 0$ frame, namely the inductor current $i_{\mathrm{Ld}}, i_{\mathrm{Lq}}, i_{\mathrm{L} 0}$, the grid-connected voltage $u_{\mathrm{od}}, u_{\mathrm{oq}}, u_{\mathrm{o} 0}$, the bridge arm voltage $u_{\mathrm{d}}, u_{\mathrm{q}}, u_{0}$ and the neutral line $i_{\mathrm{n}} . \omega$ is the grid frequency. The state variable equations in the $d q 0$ frame can be expressed as:

$$
\left\{\begin{array}{l}
u_{\mathrm{od}}=u_{\mathrm{d}}+L_{\mathrm{f}} \frac{d i_{\mathrm{Ld}}}{d t}-\omega i_{\mathrm{Lq}}+R_{\mathrm{f}} i_{\mathrm{Ld}} \\
u_{\mathrm{oq}}=u_{\mathrm{q}}+L_{\mathrm{f}} \frac{d i_{\mathrm{Lq}}}{d t}+\omega i_{\mathrm{Ld}}+R_{\mathrm{f}} i_{\mathrm{Lq}} \\
u_{\mathrm{o} 0}=u_{0}+\left(L_{\mathrm{f}}+3 L_{\mathrm{n}}\right) \frac{d i_{\mathrm{Ld}}}{d t}+R_{\mathrm{f}} i_{\mathrm{L} 0} \\
i_{\mathrm{La}}+i_{\mathrm{Lb}}+i_{\mathrm{Lc}}=i_{\mathrm{n}}
\end{array}\right.
$$

The value of $L_{\mathrm{n}}$ is small generally, whose influence can be ignored. Due to the existence of unbalanced harmonic components, it is also necessary to control the 0 -axis of the threephase four-wire converter.

\section{Controller design}

\subsection{Repetitive controller design}

The current command of grid-connected PCS is a signal containing multiple harmonics, so it is necessary to introduce a repetitive controller. This method is based on the inner membrane principle and can track the periodic repetitive control signal without static error.

The ideal repetitive controller is a unity-gain positive feedback system, and the critical stability of the system is unsatisfactory. Therefore, repetitive controller needs to be corrected and the part of its accuracy is sacrificed to ensure its stability. The structure diagram of modified repetitive controller is shown in Fig. 2.

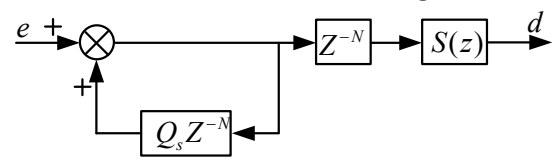

Fig. 2. The structure diagram of modified repetitive controller.

$Q$ s is the attenuation coefficient. $S(z)$ is the compensator to compensate mainly the amplitude and phase of the control system, which is mainly composed of gain compensation coefficient $k_{\mathrm{c}}$, phase compensation link $Z_{\mathrm{k}}$ and filter link $F(z)$ as follows:

$$
S(z)=k_{c} z^{k} F(z)
$$

\subsection{Compound controller design}

Considering the control of grid-connected PSC is generally current-type control method which needs to accurately track the given current signal and the slower dynamic performance of repetitive controller in the transient response, it needs to be applied in conjunction with the PI and repetitive controller. There are mainly two kinds of embedded structure and parallel type in the compound controller design method with the PI and repetitive control, as shown in Fig. 3. 


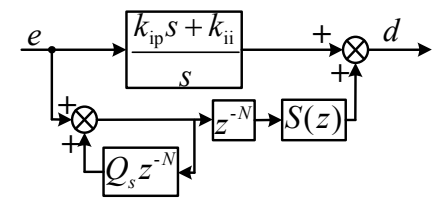

(a) Embedded compound control

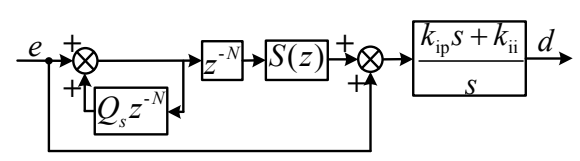

(b) Parallel compound control

Fig. 3. Compound controller block diagram with PI and repetitive control.

Embedded compound control adjusts the input error signal to make the system output value equal to the expected value. The compensation function $S(z)$ is easier to implement. But its anti-disturbance performance is not only affected by the repetitive controller, but also by the PI controller. The PI and repetitive controller of parallel compound control can respond to the error signal at the same time when the system output value is not equal to the expected value. The system can achieve stronger anti-disturbance performance under the parallel compound control.

\section{Analysis and simulation of controller}

\subsection{PCS equivalent model with compound control}

In the $d q 0$ frame, the block diagram of PCS equivalent function with compound control is shown in Fig. 4. $K_{\text {pwm }}$ is defined as the inverter equivalent gain and $T_{\mathrm{s}}$ is the time constant.

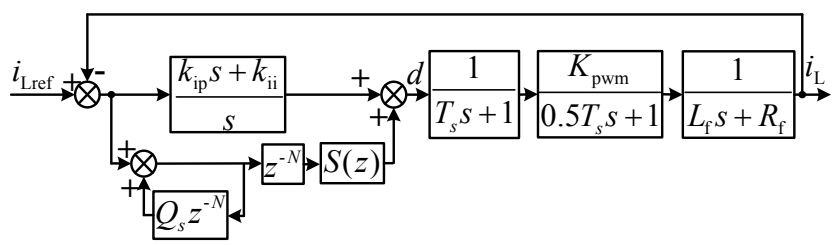

Fig. 4. Bode diagram in Case A.

$$
G_{P}(s)=\frac{\left(\frac{Z^{-N}}{1-Q_{s} Z^{-N}} S(z)+\frac{k_{\mathrm{ii}}(\tau s+1)}{s}\right) \times K_{\mathrm{pwm}}}{\left(1.5 T_{s} s+1\right) \times\left(L s+R_{\mathrm{f}}\right)}
$$

The transfer function is shown in (5), the bode diagram can be obtained by (5) to analyze the system characteristics. For the convenience, Case A is defined as the control situation that PCS achieves the best performance with only PI control. Case B is defined as the control situation that the repetitive controller is combined with the PI controller of Case $\mathrm{A}$ in parallel, which is a traditional design scheme as a comparative scheme of the proposed scheme at the same time. The simulation is achieved by Matlab/Simulink, the same frequency harmonics are injected on the grid side. The simulation parameters are given as:

Table 1. Simulation parameter.

\begin{tabular}{|l|l|l|l|}
\hline \multicolumn{1}{|c|}{ Public parameter } & \multicolumn{1}{c|}{ Value } & \multicolumn{1}{c|}{ PI controller parameter } & \multicolumn{1}{c|}{ Value } \\
\hline DC voltage $U_{\mathrm{dc}}$ & $800 \mathrm{~V}$ & Current proportional coefficient $K_{\mathrm{ip}}$ & 2 \\
\hline Switching frequency $f_{\mathrm{s}}$ & $18 \mathrm{kHz}$ & Current integral coefficient $K_{\mathrm{ii}}$ & 200 \\
\hline Rated fundamental frequency $f$ & $50 \mathrm{~Hz}$ & & \multicolumn{1}{c|}{} \\
\hline Rated phase voltage magnitude $U_{\mathrm{o}}$ & $311 \mathrm{~V}$ & Repetitive controller parameter & Value \\
\hline Filter inductor $L$ & $0.5 \mathrm{mH}$ & Attenuation coefficient $Q_{\mathrm{s}}$ & 0.95 \\
\hline Parasitic resistor of filter inductor $R_{\mathrm{L}}$ & $0.01 \Omega$ & Gain compensation coefficient $k_{\mathrm{c}}$ & 0.9 \\
\hline Filter capacitor $C$ & $20 \mu \mathrm{F}$ & Phase compensation number $k$ & 5 \\
\hline
\end{tabular}




\subsection{Model analysis and simulation}

The $G_{\mathrm{p}}(s)$ bode diagram in Case A and Case B is shown in Fig. 5.

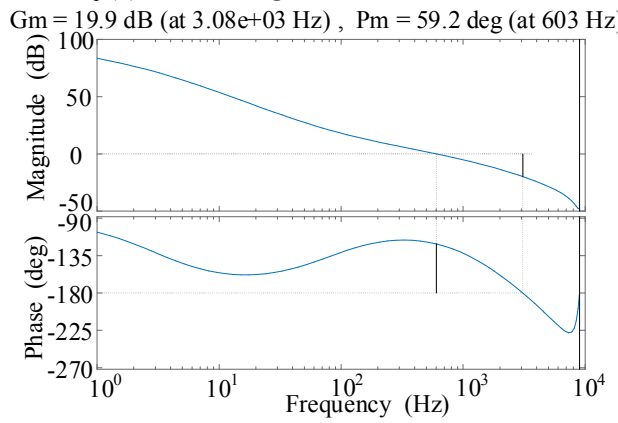

(a) Case $\mathrm{A}$

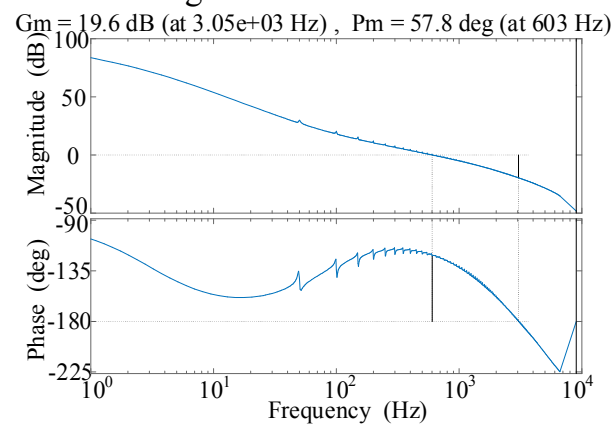

(b) Case B

Fig. 5. Bode diagram.

In Fig. 5 (a), the higher gain in low-frequency band, the amplitude-frequency characteristic passes through the $0 \mathrm{~dB}$ line with a $-20 \mathrm{~dB} / \mathrm{dec}$ slope in mid-frequency band, and the high-frequency band gain is attenuated rapidly. The both amplitude margin and phase margin are values in the best stability range for the system, indicating the PI controller parameter in Case $\mathrm{A}$ is the parameter of the best system performance, but the ability to suppress harmonics is limited. Therefore, the bode diagram in Case A that the repetitive controller is added, namely Case B, is shown in Fig. 5 (b). The system phase margin remains basically unchanged after combining the PI and repetitive controller in parallel, but the PI control gain is relatively high, which suppresses the repetitive controller gain and weakens the harmonic error compensation performance.

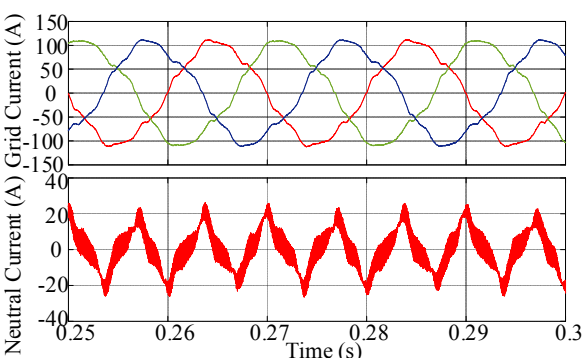

(a) Grid current and neutral current waveform in Case A

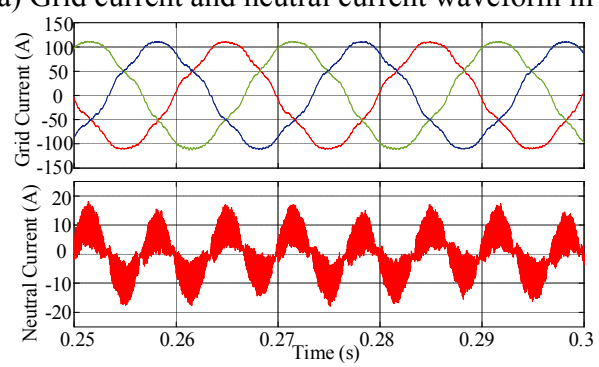

(c) Grid current and neutral current waveform in Case B

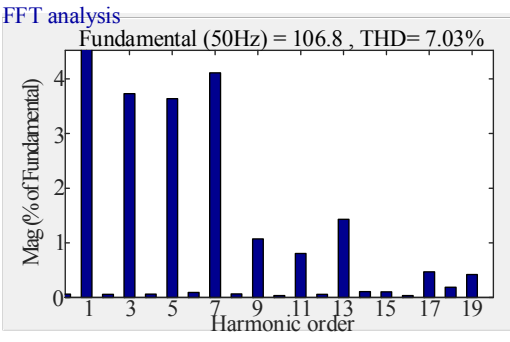

(b) Grid current THD in Case A

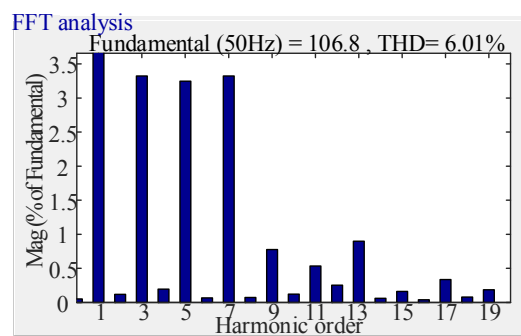

(d) Grid current THD in Case B

Fig. 6. Simulation results.

The PCS simulation results in Case A and Case B are shown in Fig. 6. Although the grid-connected PCS in Case A that only adopts PI controller can track the given signal, the grid current THD and the neutral current are too large to meet the grid-connection standard. 
The repetitive controller has a certain effect on the harmonic suppression in Case B, but the repetitive controller gain is so low that the harmonic suppression effect is unsatisfactory. The grid current THD is still greater than $5 \%$, and the neutral current is relatively large, which cannot meet the grid-connected standard.

In order to improve the harmonic suppression capability of the compound controller and ensure system stability at the same time, an optimization scheme of harmonic compensation that the repetitive control gain can be reversely increased by reducing the PI control variable gain was proposed. The PI controller parameters are changed to $K_{\mathrm{ip}}=0.5$ and $K_{\mathrm{ii}}=20$. This control situation is defined as Case C. The bode diagram in Case $\mathrm{C}$ is shown in Fig. 7.

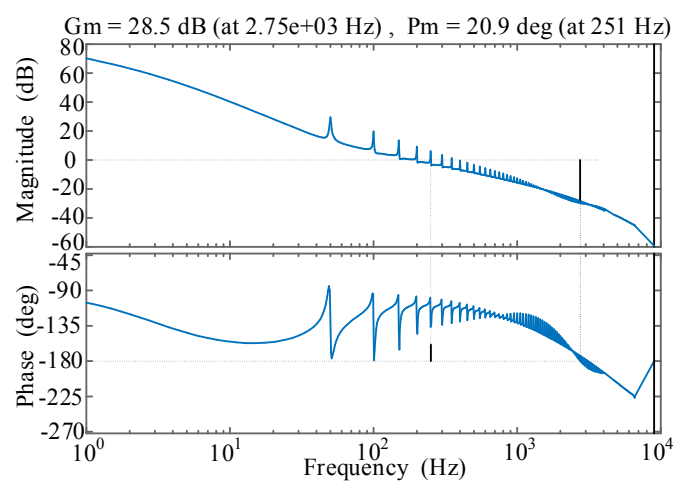

Fig. 7. Bode diagram in Case C.

In Fig. 7, the repetitive controller gain in harmonic control is increased through adjusting the gain of the PI controller, which can improve the ability of the compound controller to compensate low-frequency harmonics. The compound controller in Case $\mathrm{C}$ not only achieves the optimal control gain of the two controllers in parallel, but also ensures that the phase margin is within the stable operating range of the system. The simulation results in Case $\mathrm{C}$ are given in Fig. 8.

In Fig. 8, the grid current THD in Case $\mathrm{C}$ is reduced to less than 5\%, and the neutral current is reduced to $10 \mathrm{~A}$ without affecting the magnitude of the grid current. It is verified that the PI and repetitive control gain of the compound controller has the relationship of mutual inhibition in the harmonic current suppression.

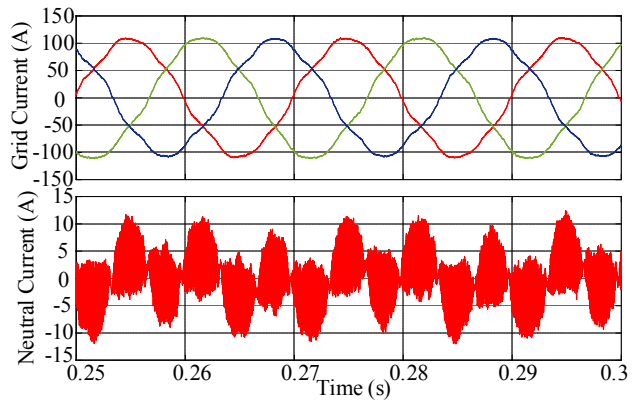

(a) Grid current and neutral current waveform

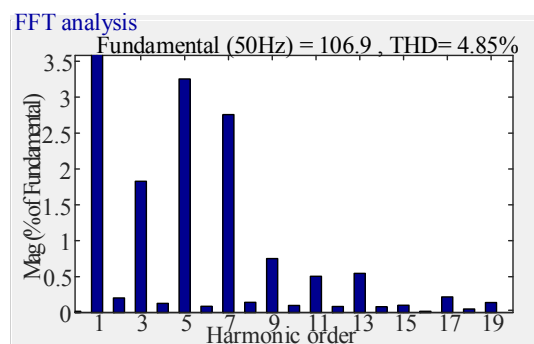

(b) Grid current THD

Fig. 8. Simulation results in Case C.

\section{Experimental results}

The experiment designed the same three situations as the simulation, namely Case A, Case 
B and Case C. The main circuit adopts the three-phase four-wire I-type three-level converter topology under the same grid-connected conditions, and the maximum power is $50 \mathrm{~kW}$. The main control chips of the experimental control circuit are composed of DSP (Digital Signal Processor) and FPGA (Field Programmable Gate Array). Different experiments are compared by adjusting the control algorithm and control parameters in the control program. The experiment waveforms of A-phase grid voltage $u_{\text {oa }}$, A-phase grid current $i_{\text {oa }}$ and neutral current $i_{\mathrm{n}}$ of PCS are shown in Fig. 9.

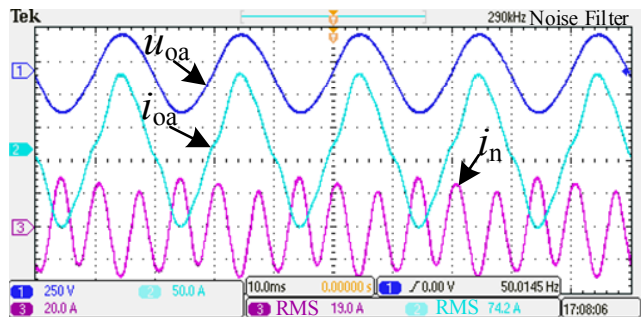

(a) Case A

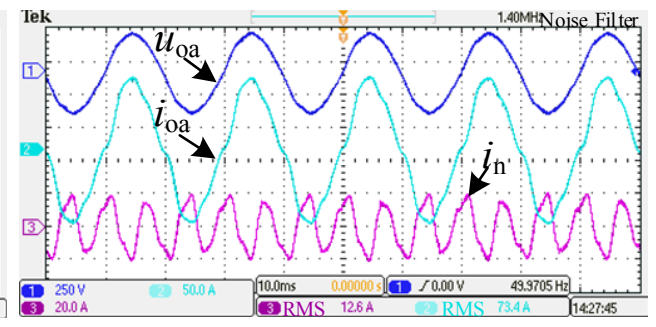

(b) Case B

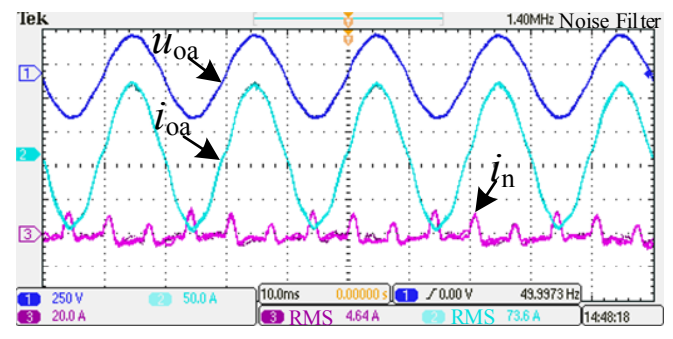

(c) Case C

Fig. 9. The experiment waveforms of A-phase grid voltage $u_{\mathrm{oa}}$, A-phase grid current $i_{\mathrm{oa}}$ and neutral current $i_{\text {n }}$ of PCS in three cases.

In Fig. 9, the experiment waveform of A-phase grid-connected voltage is the sine wave with less harmonic content under the clamping action of the grid. In Case A, the waveform quality of grid current is poor due to harmonic, and the neutral current RMS (Root Mean Square) up to 19A. The waveform quality of grid current and the amplitude of neutral current in Case B is improved slightly, but the harmonic component is still obvious and the neutral current RMS still reaches 12.6A. In Case C, the harmonic component of grid current is significantly reduced, whose waveform is close to the standard sine and the neutral current RMS is also reduced to 4.64A. The RMS of the grid current in the three cases remains almost unchanged, and the grid current THD in Case C is obviously the smallest. The experimental results prove that the harmonic suppression performance of compound controller can be improved effectively by reducing PI control gain appropriately without affecting the stability of grid current and voltage. The model analysis and simulation in the previous chapter are verified.

\section{Conclusion}

In this paper, a compound control strategy with the PI and repetitive controller in parallel is presented for the grid-connected PSC of three-phase four-wire topology. The interaction between the parameters of the compound controller is analyzed, and a controller optimization scheme is proposed. Finally, the correctness and effectiveness of the proposed scheme is verified by the simulation and experiment and draw the following conclusions: 
The compound controller of the PI and repetitive controller in parallel can have a certain effect for the harmonic current suppression of the grid-connected PCS. PI parameters will be generally designed to be the parameters which PCS achieves the best performance with only PI control, but the PI controller at this time is combined with repetitive controller in parallel, the gain of the repetitive controller will be suppressed. The compound control cannot achieve the best harmonic compensation performance. Therefore, this paper proposed that the repetitive control gain can be reversely increased by reducing the PI control variable gain. The simulation and experiment verify that the proposed scheme can improve harmonic suppression performance of compound controller and reduce the gird harmonic current of PCS without affecting the stability of gird current and voltage.

This research will further explore the compound control method for harmonic suppression in the future, and establish a complete model and optimization of control parameters to form a complete system.

\section{Acknowledgements}

This work was supported by National Natural Science Foundation of China (52077191, 51877187) and Hebei Provincial Graduate Innovative Funding Project in 2021 (CXZZBS2021139).

\section{References}

1. Y.M. Wen, L. Xiao, M.L.Y. Zheng, and Z.Q. Li, "Research on the control scheme of diode-clamped three-level inverter," Power Electron. 53, 4, 125-128 (2019)

2. Y. Cai, W. Y. Duan, and X. F. Sun, "A Novel Equivalent Infinite Active Power Filter," Acta Energi. Sin. 40, 8, 2346-2353 (2019)

3. Electromagnetic compatibility (EMC) - Part 3-4: Limits-Limitation of emission of harmonic currents in low-voltage power supply systems for equipment with rated current greater than 16 A, IEC TS 61000-3-4: Oct. 30 (1998)

4. Z. Gao, H. Zhao, X. Zhou, and Y. Ma, "Summary of power system harmonics," 2017 29th CCDC, Chongqing, 2287-2291 (2007)

5. C. Xie, X. Zhao, K. Li, J. Zou, and J. M. Guerrero, "A new tuning method of multiresonant current controllers for grid-connected voltage source converters," IEEE J. Em. Sel. Top. P. 7, 1, 458-466, March (2019)

6. N. Marati, and D. Prasad, "A modified feedback scheme suitable for repetitive control of inverter with nonlinear load," IEEE T. Power Electr. 33, 3, 2588-2600, March (2018)

7. J. Sun, J. Gong, B. Chen, and X. Zha, "Analysis and design of repetitive controller based on regeneration spectrum and sensitivity function in active power filter system," IET Power Electron. 7, 8, 2133-2140, August (2014)

8. Z. Xiao, X. Deng, R. Yuan, P. Guo, and Q. Chen, "Shunt active power filter with enhanced dynamic performance using novel control strategy," IET Power Electron. 7, 12, 3169-3181 (2014)

9. F. Liang, and Q.S. Zhao, "Application of PIRC in harmonic suppression of electric vehicle charger," Electr. Measur. Technol. 41, 6, 1-5 (2018)

10. A. Marini, L. Piegari, S-S. Mortazavi, and M.-S. Ghazizadeh, "Coordinated operation of energy storage systems for distributed harmonic compensation in microgrids," Energies, 13, 3, 771 (2020) 